Conclusion Alcohol consumption and cigarette smoking were different by gender, residence and education level in Mongolian adults.

\section{SP1-11 SELF-REPORTED HEALTH AS A PREDICTOR OF MORTALITY IN ELDERLY MEN LIVING IN SOUTHEAST BRAZIL}

doi:10.1136/jech.2011.142976m.88

L M Santiago, ${ }^{*}$ I E Mattos, L L Luz. Oswaldo Cruz Foundation, National School of Public Health, Rio de Janeiro, Brazil

Introduction Self-reported health is a perception based on an individual interpretation of physical aspects, mental status and expectations and is considered a good predictor of mortality among old people in developed countries. The aim of this study was to evaluate the role of self-reported health as a predictor of mortality in elderly men in a developing country.

Methods The study population consisted of 2875 elderly men of a medium size city in Southeast Brazil, who were followed for 4 years or until the date of their death, whichever occurred first. Individuals alive at the end of follow-up were censored. Multivariate analysis was performed through Cox regression models. Variables presenting statistically significant associations with mortality in bivariate analysis where entered into the models.

Results During the follow-up, 298 deaths occurred. Elders with selfreported poor health presented a greater risk of death, compared to those with self-reported good/excellent health in almost all stratus of the analysed variables. In the final model, poor self-reported health (HR 1.54 95\% CI 1.21 to 1.96), age (HR 1.07 95\% CI 1.06 to 1.09), marital status (HR $1.3295 \%$ CI 1.04 to 1.69), current use of cigarette smoking (HR $1.9495 \%$ CI 1.24 to 2.62), cardiovascular disease (HR 1.62 95\% CI 1.06 to 2.47), diabetes (HR $1.5395 \%$ CI 1.14 to 2.04 ) and recent hospitalisation (HR $1.5095 \%$ CI 1.15 to 1.95) were independently associated to mortality.

Conclusion Self-reported health was a good predictor of mortality in this population of elderly men, even when adjusted for other independent variables. It is important that healthcare services incorporate this indicator in the health evaluation of old people.

\section{SP1-12 RELATIONSHIP BETWEEN SLEEP DURATION AT 3 YEARS OF AGE AND GROWTH DURING CHILDHOOD: A MULTI-LEVEL ANALYSIS}

doi:10.1136/jech.2011.142976m.89

\begin{abstract}
${ }^{1} \mathrm{~K}$ Suzuki, ${ }^{* 2} \mathrm{~A}$ Takahashi, ${ }^{2} \mathrm{M}$ Sato, ${ }^{2} \mathrm{~N}$ Kondo, ${ }^{2} \mathrm{~T}$ Tanaka, ${ }^{2} \mathrm{~A}$ Nagai, ${ }^{1,2} \mathrm{Z}$ Yamagata. ${ }^{1}$ Center for Birth Cohort Studies, Interdisciplinary Graduate School of Medicine and Engineering, University of Yamanashi, Chuo, Yamanashi, Japan; ${ }^{2}$ Department of Health Sciences, Interdisciplinary Graduate School of Medicine and Engineering, University of Yamanashi, Chuo, Yamanashi, Japan
\end{abstract}

Introduction Short sleep duration in early life has been thought to be a risk factor for subsequent overweight. This study aimed to examine the relationship between sleep duration at 3 years of age and childhood weight status through a multi-level analysis.

Methods The study population comprised children born between 1 April 1991, and 31 March 2003, in Koshu City, Japan, and who participated in a medical check-up at 3 years of age. Short and long sleep durations at 3 years of age were the exposures studied. We compared the trajectory of body mass index (BMI) z-scores from 3 to 9 years of age in exposed and non-exposed participants. Random intercepts and slopes model (SAS Proc Mixed) was used for statistical analysis.
Results Of 1794 children who participated in a medical check-up at 3 years of age, 1640 (91.4\%) were not over-weight at 3 years and were followed-up until they were 9 or 10 years old. The number of children in each category of sleep duration, that is, $\leq 9 \mathrm{~h}, 9-10 \mathrm{~h}$, $10-11 \mathrm{~h}$, and $\geq 11 \mathrm{~h}$, was $66(3.7 \%), 609(34.0 \%), 847(47.2 \%)$, and $271(15.1 \%)$, respectively. BMI $z$-scores increased with increase in age $(p=0.03)$ for boys with a short sleep duration $(<9 h)$. On the other hand, sleep duration was not significantly associated with BMI z-score in girls.

Conclusion It was suggested that there is gender difference of the effect of childhood sleep duration on subsequent overweight.

\section{SP1-13 BEING BREASTFED IN INFANCY AND THE RISK OF BREAST CANCER AMONG JAPANESE WOMEN}

doi:10.1136/jech.2011.142976m.90

${ }^{1} \mathrm{Y}$ Minami, ${ }^{*}{ }^{2} \mathrm{Y}$ Nishino, ${ }^{3} \mathrm{M}$ Kawai, ${ }^{4} \mathrm{Y}$ Kakugawa. ${ }^{1}$ Division of Community Health, Tohoku University Graduate School of Medicine, Sendai, Japan; ${ }^{2}$ Division of Epidemiology, Miyagi Cancer Center Research Institute, Natori, Japan; ${ }^{3}$ Department of Surgical Oncology, Tohoku University Graduate School of Medicine, Sendai, Japan; ${ }^{4}$ Division of Surgery, Miyagi Cancer Center Hospital, Natori, Japan

Introduction The incidence of breast cancer is continuously increasing in Japan. The early life exposures such as being breastfed in infancy have been hypothesised to influence subsequent breast cancer risk

Methods We investigated the relationship between having been breastfed and breast cancer risk in a hospital-based case-control study of women aged 30 and over (573 breast cancer cases and 2155 cancer-free controls). Data on reproductive factors, lifestyle, and history of having been breastfed were collected using a selfadministered questionnaire.

Results and Conclusion After adjustment for known risk factors of breast cancer, no association for having been breastfed was observed overall (OR 1.20; 95\% CI 0.82 to 1.75). Analysis stratified according to birth year $(<1950, \geq 1950)$ demonstrated heterogeneity in the association of having been breastfed with breast cancer risk between the two birth-year groups ( $p$ for interaction $=0.0006$ ); having been breastfed was associated with a decreased risk among women who were born before 1950 (OR 0.59, 95\% CI 0.35 to 0.99), whereas it was associated with an increased risk among women born after 1950 (OR 1.65, 95\% CI 0.91 to 2.98). These findings indicate that early nutrition has some effect on breast cancer risk. In Japan, the use of standard formula supplement began to spread around 1950. Endocrine disrupters such as organochlorines were also introduced to the food chain at around the same time. The heterogeneity of breast cancer risk between the two birth-year groups may therefore be attributable to these environmental changes related to infant feeding.

\section{SP1-14 INFLUENCE OF OBESITY INDICATORS ON HYPERTENSION IN ADULTS OF RURAL INDIA}

doi:10.1136/jech.2011.142976m.91

S Anwar, ${ }^{*}$ P Deshmukh, B S Garg. Mahatma Gandhi Institute of Medical Sciences, Sevagram, Wardha, Maharashtra, India

Introduction Being overweight is associated with twofold to sixfold increase in risk of developing hypertension. Studies in urban Indian population also show this association. Present study examines relationship of different anthropometric indicators and blood pressure levels in rural population in central India about which little is known. 